\title{
The interaction of perceived distance with the perceived direction of visual motion during movements of the eyes and the head
}

\author{
MICHAEL T. SWANSTON \\ Dundee Institute of Technology, Dundee, Scotland \\ NICHOLAS J. WADE \\ Dundee University, Dundee, Scotland \\ HIROSHI ONO \\ York University, Toronto, Canada \\ and \\ KOICHI SHIBUTA \\ Kagoshima Women's College, Kagoshima, Japan
}

\begin{abstract}
A horizontally moving target was followed by rotation of the eyes alone or by a lateral movement of the head. These movements resulted in the retinal displacement of a vertically moving target from its perceived path, the amplitude of which was determined by the phase and amplitude of the object motion and of the eye or head movements. In two experiments, we tested the prediction from our model of spatial motion (Swanston, Wade, \& Day, 1987) that perceived distance interacts with compensation for head movements, but not with compensation for eye movements with respect to a stationary head. In both experiments, when the vertically moving target was seen at a distance different from its physical distance, its perceived path was displaced relative to that seen when there was no error in pereived distance, or when it was pursued by eye movements alone. In a third experiment, simultaneous measurements of eye and head position during lateral head movements showed that errors in fixation were not sufficient to require modification of the retinal paths determined by the geometry of the observation conditions in Experiments 1 and 2.
\end{abstract}

A moving target may be fixated and pursued by movements of the eyes in a stationary head, by movements of the head with the eyes stationary, or by some combination of the two. If an observer judges the direction of motion of a second target moving in a different direction to the one that is pursued, the perceived direction of motion of the second target is nonveridical and is displaced toward the direction of its motion on the retina (Becklen, Wallach, \& Nitzberg, 1984; Festinger, Sedgwick, \& Holtzman, 1976; Swanston \& Wade, 1988; Wallach, Becklen, \& Nitzberg, 1985). Compensation for the effects of eye movements on the path of retinal motion is better than that for head movements, but in both cases there is a partial failure of the processes that relate self-motion to retinal motion (Swanston \& Wade, 1988).

The outcome of such undercompensation is a perceived tilt in the path of a vertically moving target observed during a horizontal eye or head movement. A similar per-

This work was supported by NATO Grant RG $0067 / 89$ and NSERC Grant A0296. Correspondence should be addressed to M. T. Swanston, Dundee Institute of Technology, Bell Street, Dundee DD1 1HG, Scotland (e-mail:bstmts@vaxb.cc.dct.ac.uk). ceptual outcome with lateral head movements can also be caused by an error in the target's perceived distance (Gogel, 1980). In this paradigm, fixation is normally maintained on the single target during the head movements. As a result, its path of retinal motion is approximately the same as its physical motion, eliminating the effects of undercompensation described above. If the target appears farther than its physical distance, it will appear to move in the opposite direction to the head. If it appears nearer than its physical distance, it will appear to move with the head. The extent and direction of such perceived movements are predictable from the perceived distance of the target and the extent of head movement (see Gogel, 1990, for a review). In effect, an error in perceived distance acts as if the target were undergoing a real physical motion, and such perceived motions can be shown to add as vectors to real motions (Gogel, 1979, 1982). Depending on the phase relationships of vertical object motion and horizontal head motion, it is possible either to increase or decrease the apparent tilt of a vertically moving target by altering its apparent distance (Gogel, 1979). Thus, perceived distance may contribute to perceived target motion during concomitant head movements. 
In previous studies of compensation for the effects on retinal motion of eye or head movements, the tracked and judged targets have been presented, and perceived, in the same depth plane. Since the perceived distance of both targets was approximately veridical (Swanston \& Wade, 1988 ), the perceived motion path during eye and head movements would not have been differentially affected. In addition, studies of motion perceived during lateral head movements have not involved differences between the directions of retinal and physical motion of a second target. Since both undercompensation and perceived distance can be shown independently to determine perceived target motion, it is of interest to establish the extent of any interaction between the two.

A theoretical basis for predicting the form of this interaction is provided by the model that we have proposed (see Figure 1) for the recovery of information about position and motion relative to the environment (the geocentric representation) from the changing patterns of stimulation available to the eyes of a moving observer (Swanston, Wade, \& Day, 1987; Swanston, Wade, \& Ono, 1990; Wade \& Swanston, 1987). This model describes the information necessary to obtain geocentric perceptions, when the eyes move relative to the head and when the head, together with the eyes, moves relative to the environment. The initial representation of motion is with respect to the coordinates of each retina (retinocentric). Combination of the left and right monocular representations leads to a single cyclopean motion signal. If this is added to an appropriately signed signal for eye movements, a representation of motion is obtained that is relative to the head (egocentric) and is independent of

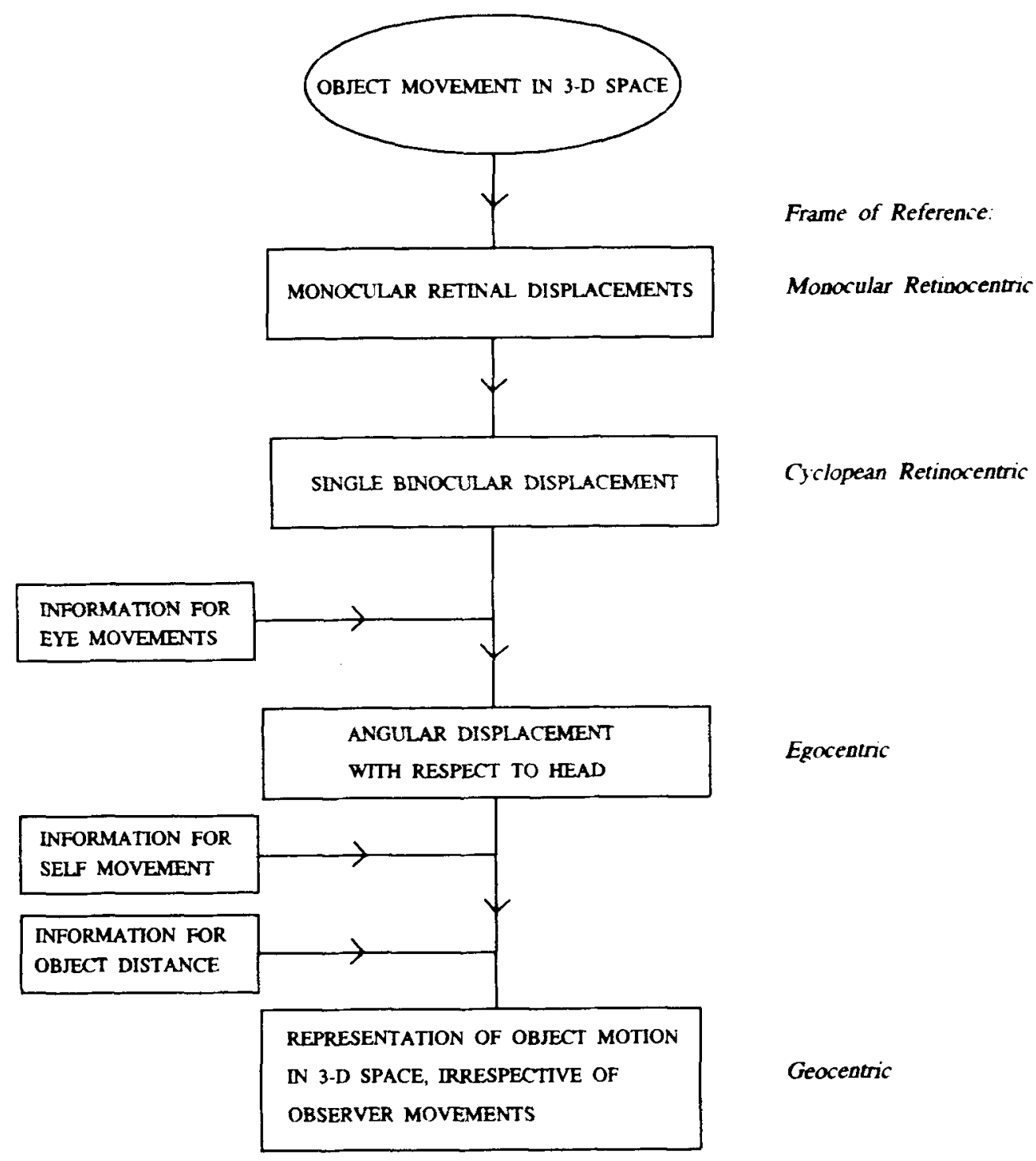

Figure 1. Outline of the model of geocentric motion perception proposed by Swanston, Wade, and Ono (1990). See text for details. 
movements of the eyes in the head. If the eyes move as the result of head movements, then such egocentric motion cannot be interpreted unambiguously unless the distance of objects from the observer is available as well. Egocentric motion can be scaled by egocentric distance to correspond to a particular extent in three-dimensional space. This scaled information is then in a form suitable for compensation for the extent of head movement, which also consists of a displacement in three-dimensional space. The model therefore predicts an interaction of perceived distance with compensation for lateral head movements, but not with compensation for eye movements. By specifying the form and sequence of the combination of various sources of spatial information, it allows prediction of the consequences of errors in the values of these. The model essentially constitutes a description of an "ideal perceiver"; the extent to which the human visual system actually achieves the necessary integration of information required for veridical perception needs to be established by experiment.

\section{EXPERIMENT 1}

The purpose of Experiment 1 was to test the prediction that the perceived path of a vertically moving target would depend on its perceived distance during head pursuit, but that this would not occur with eye pursuit. Thus, the judged target was seen as either equidistant with the pursued target or farther away. The phase relationships of the target movements were such that the apparent path with head pursuit was predicted to be more tilted when the targets appeared at different distances. In addition to the four main experimental conditions (head and eye pursuit, with targets equidistant or separated in depth), there were three control conditions. These provided measures of the perceived path without pursuit movements or changes in perceived distance, the perceived path produced during head movements while fixating a single target whose perceived distance was greater than its physical distance, and the influence of any interaction between the simultaneous motions of the two targets.

\section{Method}

Observers. Twelve paid observers took part in the experiment. All had normal or corrected-to-normal vision.

Apparatus. Stimuli were generated by an Amiga 2000 computer and were displayed with a pixel resolution of 640 (width) $\times 250$ (height) on a 35-cm-diagonal video monitor (Philips 8833). The viewing distance was $114 \mathrm{~cm}$. Observations were made in an otherwise dark room. The targets were small rectangles, 2 pixels wide $\times 1$ pixel high, subtending approximately $0.04^{\circ}$ on a side. In conditions in which there was a horizontally moving target, it moved back and forth along a path subtending $4.1^{\circ}$, with a velocity of $2.73 \% \mathrm{sec}$. A single motion from left to right, or vice versa, took approximately $1.5 \mathrm{sec}$, and there was a pause of approximately $0.5 \mathrm{sec}$ when the target reached either end of its range. In Conditions 1 and 2, this target remained stationary in the midpoint of the horizontal path and in the median plane. In Condition 3 , an additional stationary target was present, $0.25^{\circ}$ below the center of the horizontal path. The judged target moved on a vertical path located to the left of the horizontal target at a velocity of $0.8 \% \mathrm{sec}$. The vertical path subtended $1.2^{\circ}$, with its midpoint located $3^{\circ}$ from the leftmost position of horizontal path. To produce changes in the perceived distance of the judged target, two strips of oppositely oriented polaroid were applied to the surface of the screen. The single vertically moving target was replaced by two targets, moving in unison over the same path, but with an uncrossed disparity of $0.5^{\circ}$. When viewed through polaroid filters in front of each eye, this corresponded approximately to an increase in distance of $20.7 \mathrm{~cm}$, relative to the pursued target at the viewing distance of $114 \mathrm{~cm}$, and with an assumed interocular distance of $6.5 \mathrm{~cm}$. The display was viewed with the head placed on a rest, which was mounted on a track, and could be moved easily from side to side. The lateral excursion of the head was set by stops separated by $8.2 \mathrm{~cm}$ and aligned with the horizontal motion of the pursued target. A linear transducer was attached to the rest, which provided position signals during movements of the head. These signals were digitized and interpreted in real time by the software that generated the display. The experimenter's screen showed a cross that moved synchronously with the observer's head, and it provided a visual indication of the accuracy of pursuit. In addition, the signed difference between the horizontal position of the pursued target and of the observer's head was measured at 10 points approximately $0.8 \mathrm{~cm}$ apart, whenever observations were being made. After a trial, the mean difference between head and target positions was computed for each point. For conditions in which the head was not moved, the rest was locked in the center point of its travel. The judged target moved upwards synchronously with the rightward motion of the pursued target. Thus, pursuit with either the eyes or the head resulted in a retinal path tilted counterclockwise from the vertical. Given the relative extent of the vertical and horizontal motions, and assuming accurate pursuit, the retinal path was at an angle of $73.7^{\circ}$ from the vertical.

Procedure. There were seven experimental conditions (see Figure 2), each of which was seen four times by each observer, in a random order. In all conditions, the observer's task was to report the direction of motion of the left (judged) target, which always moved vertically along the same path. Initially, the different observation conditions were explained, and practice was given in the use of the chinrest for lateral head movements. Two practice trials were performed, one with eye and one with head pursuit, and a random choice of the two distances of the judged target. A check was made that observers saw the judged target as more distant when a disparity was present between the two targets. The duration of observation in each trial was determined by the observer, but was normally between 8 and 10 excursions of the judged target. On a verbal signal from the observer, a light was switched on in the observer's booth. The observer then adjusted the position of a metal rod attached to a potentiometer mounted on the vertical surface of the front of the apparatus. The angular setting was read by the experimenter from a digital voltmeter. Once this was recorded, the observer rotated the metal rod randomly away from the vertical in preparation for the next trial. For Condition 1, the head and eyes were kept stationary, fixating the stationary target, which was straight ahead and to the right of the vertically moving target. For Condition 2, the right target was again stationary, but the judged target was presented with a stereo disparity and seen as farther away. Lateral head movements were made in synchrony with the vertical movements of the judged target, and fixation was maintained on the right target. The purpose of this condition was to obtain an estimate of the perceived tilt produced only by the change in perceived distance. For Condition 3, three targets were present on the display. Two of these corresponded to the left and right targets of the other conditions. These moved vertically and horizontally, respectively. The third target was located $0.5^{\circ}$ below the center 
CONDITIONS

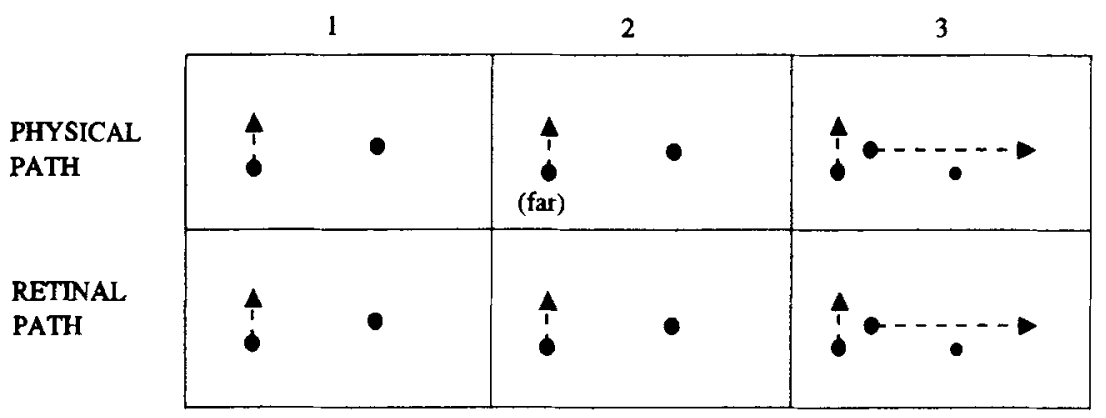

4

5

6

7

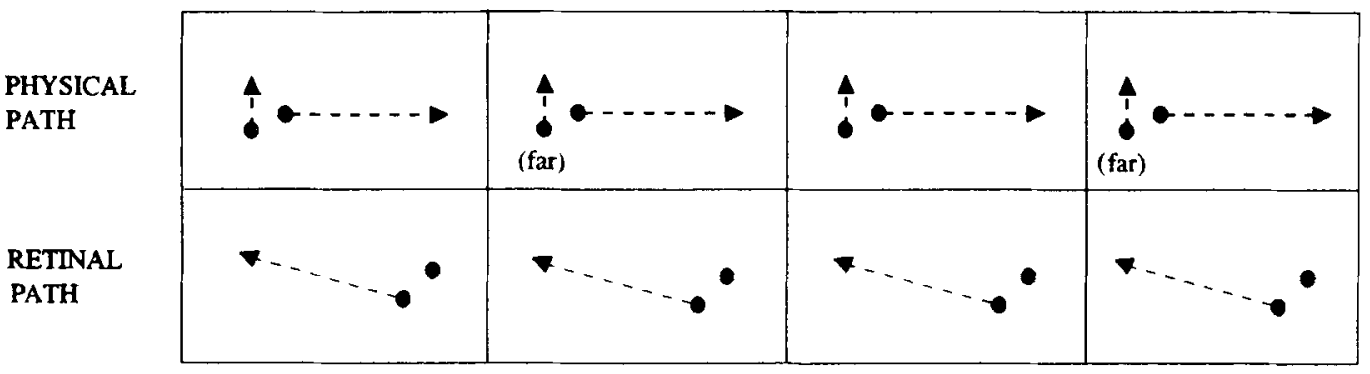

Figure 2. Schematic representation of the physical and retinal paths for each condition in Experiment 1 . In Conditions 2,5 , and 7 , the vertically moving left-hand target was presented with a stereo disparity such that it was seen as more distant than the right-hand target.

of the horizontal path of the right target, and observers maintained fixation on this point, keeping their heads stationary. This condition was designed to check for any interaction between the apparent motions of the left and right targets, which might have influenced the perceived paths independently of perceived distance or compensation for pursuit. For Conditions 4 and 5 , the chinrest was stationary in the central position, and observers pursued the horizontal motion of the right target with eye movements. For Conditions 6 and 7, the motion of the right target was followed by lateral head movements. At the start of a trial, the target and the headrest were positioned at the left of their ranges. The observer then made lateral movements synchronously with the target's horizontal motion, so as to keep the pursued target straight ahead. In Conditions 5 and 7 , the left target was presented with a stereo disparity. Figure 2 shows diagramatically the physical and retinal characteristics of the stimuli in each condition.

\section{Results}

The results of the experiment are summarized in Table 1. The values shown are the means and standard errors of the perceived paths of the judged target. The mean setting in Condition 1 was $1.0^{\circ}$ clockwise, so any bias in the judged vertical with stationary observation was slight. In Condition 3, there was a perceived counterclockwise tilt of $0.8^{\circ}$, indicating that there was no effect of interaction between the motions of the two targets. This was much less than the counterclockwise tilts observed in other conditions and also less than typical values for such interactions reported in other studies (e.g., Gogel, 1974). Swanston and Wade (1988) found no effect of this type in a similar control experiment, and it is unlikely to have played a significant part in these results. In Condition 2, there was a perceived counterclockwise tilt of $8.4^{\circ}$, as a result of the increased perceived distance of the judged target. This tilt corresponded to a horizontal motion of $0.35 \mathrm{~cm}$, relative to the vertical motion of $2.4 \mathrm{~cm}$. The perceived distance corresponding to such an apparent horizontal motion can be found from the expression (Swanston \& Gogel, 1986):

$$
D^{\prime}=D\left(K-W^{\prime}\right) / K,
$$

where $\mathrm{D}^{\prime}$ is the perceived distance, $D$ is the physical distance, $K$ is the extent of lateral head motion, and $W^{\prime}$ is the signed perceived concomitant target motion (negative

Table 1

Means and Standard Errors of Perceived Motion Paths in Experiment 1

\begin{tabular}{cccc}
\hline Condition & Target Distance & \multicolumn{1}{c}{$M$} & $S E$ \\
\hline 1 & Equal & 1.0 & 1.1 \\
2 & Far & -8.4 & 1.4 \\
3 & Equal & -0.8 & 1.4 \\
Eye Pursuit & & & \\
4 & Equal & -28.9 & 4.9 \\
5 & Far & -27.4 & 4.9 \\
Head Pursuit & & & \\
6 & Equal & -31.7 & 4.1 \\
7 & Far & -38.6 & 5.0 \\
\hline
\end{tabular}

Note-Values shown are degrees of tilt from the vertical (negative value indicates counterclockwise tilt). 
when opposite to the direction of head movement). The value obtained for Condition 2 was $119.0 \mathrm{~cm}$ (i.e., an increase in perceived distance of $5.0 \mathrm{~cm}$, relative to the physical distance of $114 \mathrm{~cm}$ ). This was approximately $25 \%$ of the distance appropriate to the stereo disparity employed, and it indicates that under these conditions disparity was not a fully effective cue or that the perceived tilt was not wholly determined by perceived distance. The perceived distance of the judged target was nevertheless increased, as shown by the change in its perceived motion path during concomitant head movements. This could in part have been due to a difference in compensation for retinal displacements due to eye and head movements. Although in Condition 2 there was no significant tilt in the retinal path of the target, the head was moved laterally while the eyes turned so as to maintain fixation on the central stationary point. Thus, the perceived path of the target depended on compensation for both eye and head movements, independently of its perceived distance. We did not find perceived tilts from this cause in our earlier work (Swanston \& Wade, 1988), but this may have been because the effect was not large enough to be measured under the conditions of that study.

The four conditions involving pursuit differed in the predicted manner. In general, as found in our earlier study (Swanston \& Wade, 1988), the perceived path during eye movements was closer to the physical path than was the case with head movements, indicating a greater degree of compensation for self-produced retinal motion in the former case. When both targets were seen as equidistant, the path with eye movements was tilted by $28.9^{\circ}$ (Condition 4), and the path with head movements was tilted by $31.7^{\circ}$ (Condition 6), both counterclockwise. However, when the stereo disparity was present, and the judged target appeared more distant, the path with eye movements remained the same (Condition $5,27.4^{\circ}$ ), while that with head movements was significantly more tilted [Condition $\left.7,38.6^{\circ}, t(11)=6.34, p<.01\right]$. Although the magnitude of the difference varied, all subjects reported a more tilted path in Condition 7 than in Condition 6 . The main prediction from the model described above was therefore confirmed. Although the difference in the tilts during head movement with and without disparity (Condition 6-Condition 7) was less than the tilt obtained in Condition $2\left(6.9^{\circ}\right.$ and $9.4^{\circ}$, respectively), this difference was not significant $[t(11)=1.0]$.

In addition, data were obtained regarding the accuracy of pursuit head movements during trials in which these were required. Each observer completed eight such trials (four with Condition 6 and four with Condition 7). After each trial, data were available for the mean signed difference between the positions of the observer's head and the pursued target at each of 10 locations across the horizontal path. Figure 3 shows the mean data for all subjects, as the error in degrees between head and target position at each location, combined for both left-to-right and right-to-left movements. The maximum error, in the middle part of the movement, was around $0.1^{\circ}$, with the head

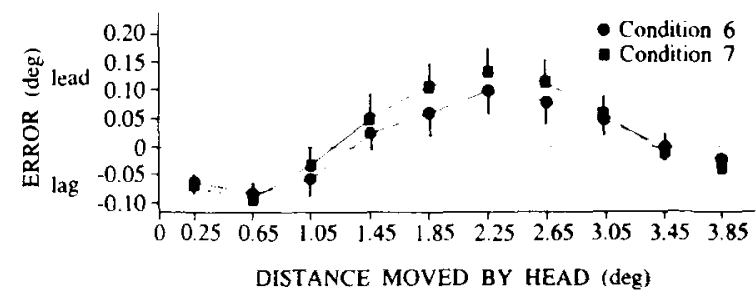

Figure 3. Mean error in head position relative to the pursued target in Experiment 1, at 10 points in the movement of the head. Lefward and rightward motions are combined. Vertical bars represent the standard error of each mean and are shown in one direction only for diagrammatic clarity.

being in advance of the target. A lag error was found only at the ends of the movement, following or preceding a stationary pause. No systematic effects on the retinal path of the judged target would be produced by such head movements, assuming that the eyes remained approximately stationary in the head. Data presented below (Experiment 3) address the issue of what, if any, eye movements may take place during such head movements. Here, subjects accelerated from rest to be somewhat ahead of the pursued target at the midpoint and then began to slow down. The pattern suggests that once the observer has become accustomed to the timing of the rhythmic head movements, they are launched ballistically from the left or right stop points rather than continuously controlled by the monitoring of visual feedback from positional errors. The pattern of errors was very similar for Conditions 6 and 7. There was perhaps a slight tendency for the head to lead the target by less in Condition 6 (equidistant targets) than in Condition 7, but the difference was on average less than $0.02^{\circ}$, and could not have produced the difference in the perceived tilts of the judged target.

\section{EXPERIMENT 2}

Experiment 1 demonstrated that the horizontal vectors of perceived motion produced by inadequate compensation for head movements and by an increase in perceived distance can combine additively to give a perceived motion path more tilted than that obtained with either of these factors in isolation. The perceived distance of the target was larger than the physical distance, so its perceived horizontal motion was against the direction of head movements. Undercompensation for head movements also results in a perceived motion against the direction of head movement, and thus the combined effect of both processes was an increased perceived tilt. By contrast, if the perceived distance of the target is less than its physical distance, the perceived horizontal motion should be in the same direction as the head movement. With a similar undercompensation for head movements, the combined result should be a less tilted motion path; that is, it should be more vertical and therefore closer to the physical path. Accordingly, measurements were made in Experiment 2 with the same procedure as in Experiment 1, but with 
crossed rather than uncrossed disparity in the critical conditions. As before, the perceived path during pursuit eye movements was expected to be unchanged by altering the perceived distance of the pursued target.

\section{Method}

Observers. Ten observers, who were paid volunteers, took part in the experiment. All had normal or corrected-to-normal vision. Eight of the observers had participated in Experiment 1.

Apparatus. The apparatus was the same as that described for Experiment 1. The polaroid filters mounted in front of each eye were reversed in orientation to give a crossed disparity when required.

Procedure. The same seven conditions were employed as for Experiment 1 . However, the judged target appeared closer than the pursued target in Conditions 2,5, and 7. The crossed disparity was $0.5^{\circ}$, which corresponded to an equivalent target distance of $98.8 \mathrm{~cm}$, or $15.2 \mathrm{~cm}$ nearer than the display screen. The observers were tested according to the same procedure, and they completed the same number of trials.

\section{Results}

Table 2 shows the results of Experiment 2. As in Table 1 , the values shown are the means and standard errors of the perceived motion paths. The results of Conditions 3, 4, and 6 broadly replicated those for Experiment 1 . There was no effect of simultaneous motion interaction (Condition 3). When the pursued and judged targets were presented in the same depth plane, the perceived tilt was larger with head movements $\left(36.6^{\circ}\right.$ counterclockwise in Condition 6$)$ than with eye movements $\left(26.6^{\circ}\right.$ counterclockwise in Condition 4). These values were close to those obtained before and indicated similar undercompensations. In Condition 2 , the perceived tilt was $5.0^{\circ}$ clockwise, as was expected for a perceived distance less than the physical distance. From Equation 1, this corresponded to a perceived distance of $111.1 \mathrm{~cm}$, or $2.9 \mathrm{~cm}$ in front of the display surface. As with the uncrossed disparity in Experiment 1, the perceived separation of the two targets was less than $25 \%$ of that simulated by the disparity. The reduced perceived distance of the judged target had no significant effect on the perceived path of the judged target during ocular pursuit in Conditions 4 and 5. However, during head pursuit with the judged target

Table 2

Means and Standard Errors of Perceived Motion Paths in Experiment 2

\begin{tabular}{ccrc}
\hline Condition & Target Distance & \multicolumn{1}{c}{$M$} & $S E$ \\
\hline 1 & Equal & -0.6 & 0.6 \\
2 & Near & 5.0 & 1.8 \\
3 & Equal & -0.9 & 0.9
\end{tabular}

Eye Pursuit

$4 \quad$ Equal $\quad-26.6 \quad 5.9$

$5 \quad$ Near $\quad-26.1 \quad 5.1$

Head Pursuit

$\begin{array}{llll}6 & \text { Equal } & -36.6 & 6.6 \\ 7 & \text { Near } & -25.5 & 5.0\end{array}$

Note-Values shown are degrees of tilt from the vertical (negative value indicates counterclockwise tilt). nearer than the pursued target, the perceived path was $25.5^{\circ}$ counterclockwise, a significantly less tilted path than that perceived with both targets equidistant $[t(9)=$ $3.58, p<.01]$. Thus, by reversing the direction of the difference between physical and perceived distance, the effect on the perceived motion path was also reversed. Taken together, these results provide confirmation for the predictions made from our model for the perception of geocentric motion. As in Experiment 1, the tilt in Condition 2 was not equivalent to that given by the difference between Conditions 6 and 7, although in principle both were indirect measures of perceived distance. While the difference between these measures did not reach significance $[t(9)=1.51]$, the discrepancy found in both experiments suggests caution in generalizing measures of perceived distance from concomitant head movement across displays with different numbers of visible elements.

Data were also obtained for the accuracy of head pursuit. The pattern of results was almost identical to that for Experiment 1. An apparently ballistic movement resulted in a lag at either end of the horizontal range, followed by a period in which the head led the target in the middle section. The positional error was within $\pm 0.15^{\circ}$.

\section{EXPERIMENT 3}

Data obtained in Experiments 1 and 2 showed that the position of the head during pursuit of a horizontally moving target was close to the position of the target. However, eye position was not measured at the same time, so it remained possible that the observers' eyes might have moved in such a way as to invalidate the assumptions made about the path of retinal motion. For example, the eyes might have turned so as to maintain fixation on the point being fixated before the head movement began. If so, a different explanation would be required for the perceived path of a vertically moving target. Under other conditions, such a turning of the eyes would be expected, in the form of the vestibulo-ocular reflex (VOR). The VOR is typically obtained following rotation, rather than lateral displacement, of the head (Howard, 1982), and a moving target would not be present. However, the possibility that some effect of this type might occur was considered sufficiently important to warrant an attempt to make simultaneous measurements of head and eye position during head pursuit.

\section{Method}

Observers. Three observers, all of whom were authors (M.T.S., N.J.W., and K.S.), took part in the experiment.

Apparatus. The apparatus was located in a different laboratory, but was set up to match as closely as possible the apparatus employed in Experiments 1 and 2. Stimuli were generated by a Commodore Amiga 2500 and presented on a Commodore 1084 video monitor. There were two stimulus patterns. One, for calibration purposes, consisted of three stationary points, $2.05^{\circ}$ apart horizontally, in positions corresponding to the left, right, and center positions of the path of the pursued target. For the other, a single point moved back and forth along a horizontal path subtending $4.1^{\circ}$, at a velocity of $1.75^{\circ} / \mathrm{sec}$. This was somewhat less than the velocity 
employed previously. The observers viewed the display from $114 \mathrm{~cm}$. A headrest with a bite board was mounted on a track, and it activated a potentiometer as it was moved from left to right through a distance of $8.2 \mathrm{~cm}$. An eye-position sensor (Biometrics SGH/V2) was mounted on a rigid frame attached to the headrest. The sensor was adjusted for maximum sensitivity when the observer gripped the bite board with his head on the rest. Signals from the eye-position sensor and from the potentiometer were recorded on an FM tape recorder and displayed on a Beckman Type $\mathbf{R}$ Dynograph. In addition, the computer generated a marker signal at the start and end of a target movement, and this was also recorded. The apparatus was constructed so that observations were made from a standing position.

Procedure. Each observer completed a session of testing that consisted of calibration, followed by head pursuit and a final calibration. Calibration was obtained by presenting the three stationary targets, with the headrest in the central position. The observer fixated the central, left, central, and right targets in turn, twice. For head pursuit, the target moved repeatedly from left to right and back, and the observer followed this movement by lateral movements of his head, while maintaining fixation on the target. Ten cycles of target movement were pursued.

\section{Results}

The main purpose of Experiment 3 was to establish whether there was any tendency to move the eyes during a head movement, which would result in maintaining fixation on the point in the straight-ahead position before the start of the head movement. The first three pursuit cycles were discarded to allow for practice. Data were examined from the following three pursuit cycles, which for each subject provided stable measurements. Head position with respect to the target was found by plotting a line representing target position over the record for head position. At each of 12 positions in the cycle, approximately $0.5 \mathrm{sec}$ apart (equivalent to $1.6 \mathrm{~cm}$ or $0.8^{\circ}$ of linear movement), a measurement was made of the extent to which the observer's head was to the left or right of the target. For left-to-right target movements, a leftward position of the head represented a lag error, and vice versa. Eye position was recorded with respect to the head. For these data, the deviation to the left or right of the straight ahead (obtained from calibration trials) was found at the same twelve locations. This procedure effectively eliminated saccadic eye movements from the mean data. These provided a measure of the direction of gaze over the course of a head movement, as was required to establish whether eye movements could have altered the retinal path of the judged target from that expected from the extent of the head movement. The mean data for the 3 subjects for head and eye position are plotted in Figure 4 . The actual measurements of eye position obtained in the experiment correspond to the separation of the head and eye data points. Head pursuit errors were somewhat larger in Experiment 3 than in Experiments 1 and 2, but were still within about $0.25^{\circ}$ of the target. There was a tendency for the observers to undershoot the rightmost target location, but to be close to the correct position at the leftmost point. The data indicate that the head was typically ahead of the target, by approximately $0.2^{\circ}$ on average. Eye position with respect to the target clearly follows that of the head

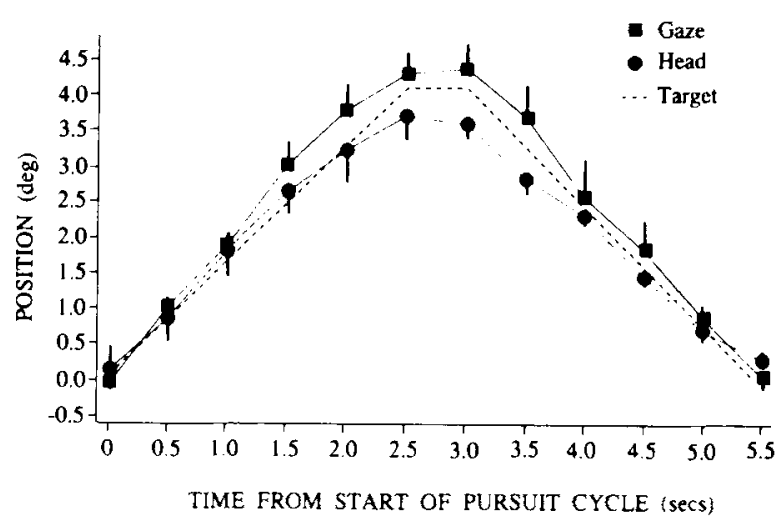

Figure 4. Mean position of the head and gaze relative to the pursued target in Experiment 3. Head movements were rightward initially, followed by a 0.5 -sec pause, and a leftward return to the initial position. Vertical bars represent the standard error of each mean and are shown in one direction only for diagrammatic clarity.

and generally seems to reflect overcorrection of errors in head position. There is no indication of eye movements to maintain fixation on the initial location of the target before the start of a target movement. Within the limits of this experiment, it can be concluded that eye movements during head pursuit did not produce a nonlinear retinal path of target motion. Ideally, eye-movement data would have been obtained from naive subjects during the course of Experiments 1 and 2. However, this would have been impracticable, given the demands imposed on observers in recording eye movements. These data can therefore only be applied to the interpretation of the earlier experiments with caution. However, head-movement recordings were available from the earlier experiments, and they showed smaller tracking errors than those obtained here. It is therefore likely that any eye movements by observers would also have been smaller, since their function during head movements appears to be to maintain fixation on the pursued target.

One issue raised by these data concerns the difference in the tilt of the path of the judged target with head and eye pursuit found in Experiments 1 and 2. The eyes rotated by about $0.6^{\circ}$ to the right during a left-to-right head movement, and the reverse $\left(0.6^{\circ}\right.$ to the left $)$ as the head moved back to the left. This would have produced a more tilted retinal path for a vertically moving target than would be expected if the eyes had remained stationary. In effect, a further horizontal vector was added to the motion. An eye rotation of $0.6^{\circ}$ during head movement would have produced a retinal path for the judged target in $\mathrm{Ex}$ periments 1 and 2 of $75.4^{\circ}$ rather than $73.7^{\circ}$, a difference of $1.7^{\circ}$. The difference in perceived tilt for eye and head pursuit was however between $5^{\circ}$ and $10^{\circ}$, so eye rotation of the magnitude observed here would not have been sufficient to account for the result. In addition, the data for head-pursuit accuracy in Experiments 1 and 2 did not indicate a tendency to undershoot the rightmost target position, which may have been caused in Experiment 3 
by the greater difficulty of making head movements while using a bite board and with the eye position sensors in place.

\section{DISCUSSION}

The results of these experiments confirm previous findings that a target that moves in a different direction from the eyes is perceived to move in a direction displaced toward the retinal path of motion. We interpret this as a consequence of undercompensation for the effect of eye movements on the retinal motion of objects. Such undercompensation is greater when the eyes move as result of a lateral motion of the head, relative to their rotation with respect to a stationary head. In addition, Experiments 1 and 2 provide information regarding the role of perceived distance in the perception of motion with a moving head. Objects viewed during concomitant lateral head movements appear to move themselves if their perceived distance differs from their physical distance (Gogel, 1980). The extent of this perceived object motion is proportional to the magnitude of the error in perceived distance. It is opposite to the direction of head movement when perceived distance is larger than the physical distance, and in the same direction as the head movement when the perceived distance is less than the physical distance. In effect, it constitutes a perceived motion vector that adds to any physical motion (Gogel, 1982). Our experiments demonstrate that the perceived path of motion of a target moving in a different direction to the head is determined both by undercompensation for effects of head movements on the target's retinal path and by the target's perceived distance. These two factors contribute vectors to the perceived motion path, which increased the apparent tilt in Experiment 1 and decreased it in Experiment 2, as the result of changing the perceived distance from farther to nearer than the physical display. Perceived distance had no effect on the perceived path of motion when the eyes were rotated in a stationary head. This pattern of results is predicted by our model of motion perception (Swanston et al., 1987), which identifies the sources of information required for veridical motion perception, and the manner of their combination. We have argued that observers' reports are based on the geocentric representation. Thus, a given retinal path is interpreted in terms of the available information regarding eye movements, head movements, and perceived distance. In the conditions involving eye movements, the perceived path of the target was not determined by perceived distance, because this would have influenced both the vertical and the horizontal components of the motion equally.

In Experiments 1 and 2, the perceived distance of the judged target as measured by its concomitant motion during head movements was considerably less than that simulated by the disparity present in the display. It could be argued that the perception of the disparity as a small depth interval indicated that the perceived distance of the display was markedly less than its physical distance, since the perceptual effect of a given disparity is scaled by perceived distance. If this were so, it could also account for a difference between the perceived tilts with eye and head pursuit, even in the absence of disparity. However, Swanston and Wade (1988) found a similar difference in perceived tilt with the two types of pursuit and demonstrated by a head-movement procedure that physical distance and perceived distance were the same in an equivalent display. More probably, the relative ineffectiveness of the disparity was due to the off-center observation of the target and to its having been viewed during lateral head movements.

Experiment 3 showed that the movements of the eyes and/or head were not systematically different from the motion of the pursued target. While errors of pursuit were present, these were not such as to give a retinal path of the judged target either more or less similar to the perceived path. It appears reasonable to assess the extent of compensation for eye or head movements on the basis of a retinal path derived from accurate pursuit, at least to a first approximation. Data for the accuracy of head movements obtained in Experiments 1 and 2 support this conclusion, as does the comparison of these results with those of our previous study (Swanston \& Wade, 1988). In the experiments described here, a pursuit procedure was employed in which observers followed the horizontal movements of the pursued target in the same manner for both head and eye movements. In our previous study, the motion of the judged target was linked to the motion of the head, but this was not the case for pursuit eye movements. In part, this was intended to ensure that the retinal path during head movements was predictable in the absence of information for the accuracy of head movements. Since the outcome in comparable conditions was the same in both studies, it is likely that head movements are the same both when the target is locked to head position and when it is followed voluntarily.

In general, the data reported here add to evidence for the "phenomenal geometry" advanced by Gogel (1990). From this viewpoint, the perceived characteristics of objects (e.g., size, shape, and motion) are determined by internal values for retinal extents, the movements of the eyes with respect to the head, the distance of the object, and motion of the self in three-dimensional space. The combination of these values follows rules that reflect the geometry of Euclidean space, and the outcome is a perceptual representation that can act as a reliable guide to action. Errors in these internal values give rise to predictable and interrelated errors in perception.

\section{REFERENCES}

Becklen, R., Wallach, H., \& NitzberG, D. (1984). A limitation of position constancy. Perception \& Psychophysics, 10, 713-723.

Festinger, L., Sedgwick, H. A., \& Holtzman, J. D. (1976). Visual perception during smooth pursuit eye movements. Vision Research, 16, $1377-1386$.

Gogel, W. C. (1974). Relative motion and the adjacency principle. Perception \& Psychophysics, 26, 425-437. 
GoGEL, W. C. (1979). The common occurrence of errors of perceived distance. Perception \& Psychophysics, 25, 2-11.

Gogel, W. C. (1980). The sensing of retinal motion. Perception \& Psychophysics, 28, 155-163.

GOGEL, W. C. (1982). Analysis of the perception of retinal motion concomitant with a lateral motion of the head. Perception \& Psychophysics, 32, 241-250.

Gogel, W. C. (1990). A theory of phenomenal geometry and its applications. Perception \& Psychophysics, 48, 105-123.

Howard, I. P. (1982). Human visual orientation. New York: Wiley. Swanston, M. T., \& Gogel, W. C. (1986). Perceived size and motion in depth from optical expansion. Perception \& Psychophysics, 39, 309-326.

Swanston, M. T., \& Wade, N. J. (1988). The perception of visual motion during movements of the eyes and of the head. Perception \& Psychophysics, 43, 559-566.
Swanston, M. T., Wade, N. J., \& Day, R. H. (1987). The representation of uniform motion in vision. Perception, 16, 143-160.

Swanston, M. T., WAde, N. J., \& Ono, H. (1990). The binocular representation of uniform motion. Perception, 19, 29-34.

WadE, N. J., SWANSton, M. T. (1987). The representation of nonuniform motion: induced movement. Perception, 16, 555-571.

Wallach, H., Becklen, R., \& Nitzberg, D. (1985). The perception of motion during colinear eye movements. Perception \& Psychophysics, 38, 18-22.

(Manuscript received September 12, 1991; revision accepted for publication June 4, 1992.) 